\title{
Second-parameter Globular Clusters in the Milky Way and in M33 as Tracers of Mass Loss from M31 in the Early Epoch?
}

\author{
Valery V. Kravtsov \\ Sternberg Astron. Ins., 13, University Ave., Moscow 119899, Russia
}

\begin{abstract}
I suggest that the bulk of the "young", second-parameter globular clusters (SPGCs) observed in the outer halo of our Galaxy and recently found in the other massive spiral of the Local Group (LG), M33, may have originated due to mass outflow from M31 and subsequent accretion of gas on the Galaxy and M33 in the early epoch.
\end{abstract}

\section{Problem and Main Questions}

There are contradictory conclusions about the dwarf spheroidal (dSph) galaxies' tidal dissolution and its rate, as well as a number of observational constraints on this process in the Galaxy (e.g., van den Bergh 1996; Gilmore et al. 1999; Shetrone et al. 2001). Moreover, preliminary results on the CMD study of GCs in massive spirals of the LG suggest a fraction of SPGCs to be at least low among GCs in M31 (Rich et al. 2002) and to be high as for the M33 GC population (Sarajedini et al. 1998). Hence there appear doubts about tidal disruption/stripping of dSphs as universal mechanism of and main contributor to a formation of populations of the SPGCs in the above three galaxies. Note also that Chandar et al. (2001) have estimated the total number of GCs in M 33 to be $75 \pm 14$ which gives a specific frequency significantly higher than in other late-type spirals.

By now, it is established that the Galactic population of SPGCs shows a number of basic distinctive characteristics compared with those of other GCs in the halo of the Milky Way (e.g., Da Costa 1994; van den Bergh 1996). Some of these characteristics imply that the Galactic SPGCs are very likely to have been accreted. However, if the tidal dissolution of dSphs is not the main and the only contributor to the formation of population(s) of the SPGCs in our Galaxy (and in M33) then the following questions arise: What is the accreted material and where did it originate and come from? Why has the formation of SPGCs lasted after the formation of the bulk of the halo objects in rapid collapse?

\section{Putative Role of the Mass Outflows}

The high-redshift Lyman break galaxies (LBGs) are suggested to be progenitors of the present-day massive spheroids, being in particular components of luminous early-type spirals (Friaca \& Terlevich 1999). As summarized by Heckman (2000) starburst-driven galactic superwinds observed in LBGs play an important role 
in the mass outflow from them. During the most powerful episode $(\sim 2 \mathbf{G y r})$ of formation of the Pop. II stars in a star-forming galaxy like M31, its outflows may remove a total gas mass as large as the mass of the stellar bulge and spheroid, with typical speeds ranging from a few $10^{2}$ to $1^{3} \mathbf{k m ~ s}{ }^{-1}$ (Pettini et al. 2001). A few tens of percent of this mass $\left(\Delta \mathbf{M} \sim \mathbf{1 0}^{\mathbf{1 1}} \mathbf{M}_{\odot}\right.$ or so) with velocities exceeding the escape velocity of M31 could have left the galaxy, some of which might have been accreted by our Galaxy (and M33). If we accept the present distance between the Galaxy and M31 ( 0.7 Mpc), the ratio of their mass $\left(\mathbf{M}_{\mathbf{G}} / \mathbf{M}_{\mathbf{A}} \sim \mathbf{1} / \mathbf{1 . 5}\right)$, and the mean velocity of the expanding gas to be of order $400 \mathrm{~km} / \mathrm{s}$, then we easily find that a time spent by gas to reach a region of the Lagrangian point between the two galaxies, may be as long as $\sim \mathbf{1} \mathbf{G y r}$. An upper limit of mass $\delta \mathrm{M}$ of gas accreted on the Galaxy is estimated from a formula obtained (in the approach of gas accretion in gravity potential $\varphi \sim R^{-1}$ of a field of less massive companion in a binary system) and kindly offered by Postnov (2000, private communication):

$$
\delta M \sim \frac{1}{4}\left(\frac{M_{G}}{M_{A}}\right)^{2} \Delta M \sim \frac{1}{10} \Delta M \sim 10^{10} M_{\odot}
$$

Even if a mass of really accreted gas was an order of magnitude lower than the upper limit estimated and if in turn approximately ten percent of this mass was converted into objects, i.e., $\sim \mathbf{1 0}^{\mathbf{8}} \mathbf{M}_{\odot}$, that would be equal to a total mass of the bulk of SPGCs, of a few dSphs, and of some portion of field stars which may have formed due to such a process in the outer halo of our Galaxy.

Similarly, it is possible to conclude that objects assumed to form from a gas accreted onto M33 might comprise a total mass of order a few $1^{6} \mathbf{M}_{\odot}$. Some 10 to 20 GCs (with a fraction of the field stars formed) with cluster's typical mass of order $10^{5} M_{\odot}$ may amount to just this mass.

Acknowledgments. I am deeply thankful for the IAU travel grant. Also, I am very grateful to Dr. A.A. Suchkov for his encouraging and valuable comments on the scenario presented, and to Drs. K. Postnov, Yu.A. Shchekinov, Yu.N. Efremov, N.I. Shakura, and M.V. Sazhin for helpful discussions.

\section{References}

Chandar, R., Bianchi, L., \& Ford, H. C. 2001, A\&A, 366, 498

Da Costa, G. S. 1994, In ESO Conference and Workshop Proc., 51, The Local Group: Comparative and Global Properties, ed. A. Layden, R.C. Smith \& J. Strom, 101

Friaca, A. C. S. \& Terlevich, R. J. 1999, MNRAS, 305, 90

Gilmore, G., Hernández, X., Valls-Gabaud, D. 1999, astro-ph/9910409

Heckman, T. M. 2000, astro-ph/0009075

Pettini, M., Steidel, C. C., Shapley, A. E., et al. 2001, ApJ, 554, 981

Rich, R. M., Corsi, C. E., Cacciari, C. et al. 2002, this volume

Sarajedini, A., Geisler, D., Harding, P., \& Schommer, R. 1998, ApJ, 508, L37

Shetrone, M., Cote, P., Sargent, W. L. W. 2001, ApJ, 548, 592

van den Bergh, S. 1996, PASP, 108, 986 